\title{
ELECTRICAL PROPERTIES OF InGaP DOPED WITH Si
}

\author{
E. Litwin-Staszewska, W. Trzeciakowski, R. Piotrzkowski \\ High Pressure Research Center, Polish Academy of Sciences \\ Sokołowska 29/37, 01-142 Warsaw, Poland
}

and L. Gonzalez

Instituto de Microelectronica de Madrid, CSIC, 28760 Tres Cantos, Spain

We measured Hall concentration $n$ in InGaP:Si epitaxial layers grown by MBE as a function of pressure $P$ up to $2 \mathrm{GPa}$ and of temperature $T$ from 77 to $300 \mathrm{~K}$. We interpreted our results in terms of the broad distribution of impurity states resonant with the conduction band. From the low-temperature $n(P)$ dependence we can directly obtain the total density of impurity states around the Fermi level $\rho\left(E_{\mathrm{F}}\right)$. The Fermi level can be shifted with respect to impurity states by applying pressure and by using samples with different $n$. In this way we obtain $\rho(E)$ in a wide energy range. We discuss the possible reasons for the observed broad distribution of $\rho(E)$.

PACS numbers: 72.20.Fr, 72.80.Ey, 71.55.Eq

InGaP lattice matched to GaAs (i.e. with $48 \%$ of indium) is an important material for red-emitting laser diodes. For the MBE growth of $n$-type InGaP the $\mathrm{Si}$ donor is commonly used. However, there is very little data in the literature concerning the electrical properties of this material. We have studied epitaxial layers (around 1 micron thick) of InGaP:Si grown by ALMBE and MBE on semi-insulating GaAs. Hall concentration $n$ in our samples (at $T=300 \mathrm{~K}$ ) varied from $2 \times 10^{17} \mathrm{~cm}^{-3}$ up to $5 \times 10^{18} \mathrm{~cm}^{-3}$. In this range we are above the Mott transition and we can neglect the effect of surface states (it is believed that InGaP has a much lower density of surface states as compared to AlGaAs). We measured $n$ and mobility $\mu$ as functions of pressure $P$ (up to $20 \mathrm{kbar}$ ) and temperature $T$ (from 20 to $300 \mathrm{~K}$ ) using the gas cell up to $1.2 \mathrm{GPa}$ and the liquid cell for higher pressures. The temperature variation of electron concentration was rather weak and similar to the literature data interpreted as due to the ionization of shallow-impurity levels [1]. However, the pressure variation of Hall concentration was substantial. This definitely eliminates the shallow-impurity interpretation. In Fig. 1 we show the $n(P)$ dependence at $77 \mathrm{~K}$ for three samples. As we can see, the concentration drops for each sample. The decrease in the electron concentration 


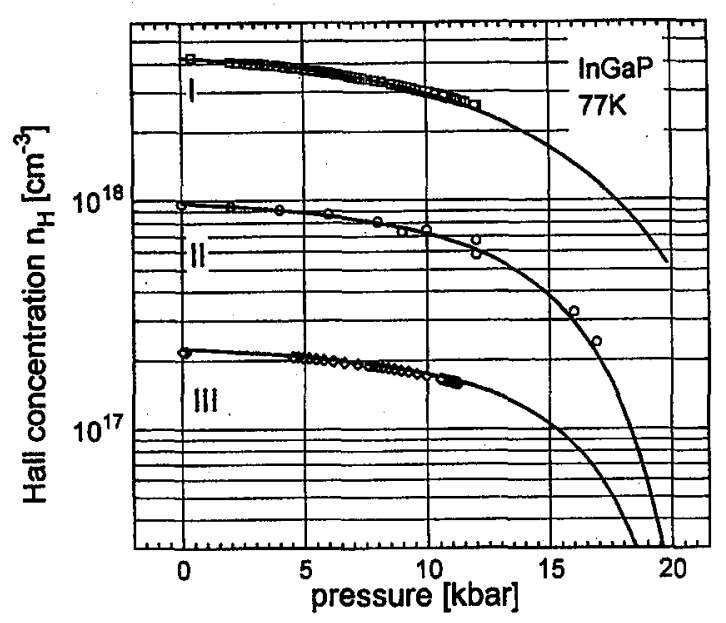

Fig. 1. Electron concentration vs. pressure for three different InGaP:Si samples. Theoretical curves have been obtained using the Lorentzian density of resonant states centered at $216 \mathrm{meV}$ above the conduction-band edge, half-width $42 \mathrm{meV}$. Sample I: $N_{\mathrm{d}}=8.3 \times 10^{18} \mathrm{~cm}^{-3}, N_{\mathrm{a}}=3.2 \times 10^{18} \mathrm{~cm}^{-3}$. Sample II: $N_{\mathrm{d}}=3.45 \times 10^{18} \mathrm{~cm}^{-3}$, $N_{\mathrm{a}}=2.22 \times 10^{18} \mathrm{~cm}^{-3}$. Sample III: $N_{\mathrm{d}}=0.91 \times 10^{18} \mathrm{~cm}^{-3}, N_{\mathrm{a}}=0.61 \times 10^{18} \mathrm{~cm}^{-3}$.

with pressure must be due to some impurity states resonant with the conduction band. Very similar $n(P)$ dependences were observed at $300 \mathrm{~K}$. This similarity indicates that we must be dealing with some slowly varying tail of a broad distribution of impurity states $\rho(E)$. For a sharp resonance in the conduction band we would obtain a slow decrease in $n(P)$ at $300 \mathrm{~K}$ and a sharp drop at $77 \mathrm{~K}$.

The standard analysis of $n(P)$ consists in assuming a certain shape of $\rho(E)$ (typically Gaussian or Lorentzian) centered at the energy $E_{\mathrm{d}}$ and having the width $\Gamma$. This density is proportional to the total donor concentration $N_{\mathrm{d}}$ larger than the electron concentration at $P=0$ (because of compensation and because some electrons may already be bound at $P=0$ ). In the case of $E_{\mathrm{d}}$ lying high in the conduction band and the broad distribution $\rho(E)$ the parameters $E_{\mathrm{d}}, \Gamma$, and $N_{\mathrm{d}}$ are interdependent because $n(P)$ is mainly sensitive to $\rho(E)$ around the Fermi level (i.e., to the tail of $\rho(E)$ and the same tail may be obtained for a different set of $E_{\mathrm{d}}, \Gamma$, and $N_{\mathrm{d}}$ ). Therefore, in the present paper we developed a different approach allowing for the direct determination of $\rho(E)$ from $n(P)$ measured at low temperature.

We start from the condition of charge neutrality in the sample

$n+n_{\mathrm{b}}=N_{\mathrm{d}}-N_{\mathrm{a}}$,

where $N_{\mathrm{a}}$ is the acceptor concentration and $n_{\mathrm{b}}$ is the concentration of electrons bound on donors given by

$$
n_{\mathrm{b}}=\int_{-\infty}^{+\infty} \frac{N_{\mathrm{d}} \rho(x) \mathrm{d} x}{1+(1 / g) \exp \left(x+E_{\mathrm{d}}-E_{\mathrm{F}} / k T\right)},
$$

where $g$ is the degeneracy of the state, $E_{\mathrm{F}}$ is the Fermi energy, and $\rho\left(E-E_{\mathrm{d}}\right)$ is the density of impurity states (per one impurity) centered at $E=E_{\mathrm{d}}$. Equations (1) 
and (2) are valid for donors that bind one electron. For the DX-like donors that can bind two electrons due to the lattice-relaxation effects the above equations are still valid if we replace $k T$ by $k T / 2$ and $N_{\mathrm{d}}$ by $2 N_{\mathrm{d}}$. The degeneracy factor can then be taken as 4 .

Now we differentiate Eq. (1) with respect to pressure and we evaluate $\mathrm{d} n_{\mathrm{b}} / \mathrm{d} P$ using Eq. (2). If we assume that $\rho(E)$ shifts with pressure without changing its shape then the integrated function will contain $\rho(E)$ multiplied by the energy derivative of the Fermi distribution (localized within a few $k T$ around $E_{F}$ ). We can expand $\rho(E)$ around $E_{\mathrm{F}}$ and perform the integration. Only the even terms of the expansion contribute to the integral. Retaining only the first two terms (which is valid if the variation of $\rho(E)$ around $E_{\mathrm{F}}$ is slow in the $k T$ range) we obtain

$$
\frac{\mathrm{d} n}{\mathrm{~d} P}=N_{\mathrm{d}} \frac{\mathrm{d}\left(E_{\mathrm{d}}-E_{\mathrm{F}}\right)}{\mathrm{d} P}\left[\rho\left(E_{\mathrm{F}}-\widetilde{E}_{\mathrm{d}}\right)+\rho^{\prime \prime}\left(E_{\mathrm{F}}-\widetilde{E}_{\mathrm{d}}\right) \frac{(k T \pi)^{2}}{6}\right],
$$

where $\widetilde{E}_{\mathrm{d}}=E_{\mathrm{d}}-k T \ln g$. At low temperature the second term can be neglected and Eq. (3) allows us to determine the total density of impurity states around the Fermi level in terms of the measured $\mathrm{d} n / \mathrm{d} P$. The above equation has a simple physical interpretation: the electron concentration in the band changes with pressure when we have a non-zero density of impurity states at the Fermi level and when the distance $E_{\mathrm{d}}-E_{\mathrm{F}}$ changes with pressure. The advantage of this approach in comparison with the standard analysis is that we obtain $\rho(E)$ without any assumptions about its shape and without using interdependent parameters $N_{\mathrm{d}}, E_{\mathrm{d}}$, and $\Gamma$. Equation (3) is valid under two assumptions: (i) that $\rho(E)$ is slowly varying in the region $\left[E_{\mathrm{F}}-k T, E_{\mathrm{F}}+k T\right]$, (ii) that $\rho(E)$ shifts with pressure without changing its shape. The first assumption is justified when the Fermi level scans the tail of $\rho(E)$ and it works better at low temperature. If the observed changes of $n(P)$ at $77 \mathrm{~K}$ are slow this assumption should be fulfilled. The second assumption depends on the physical origin of the broadening of $\rho(E)$. If the impurity states are smeared by the fluctuations of composition of the alloy or by the alloy splitting of impurity states then this assumption is justified. In the case when the broadening of $\rho(E)$ is due to potential fluctuations originating from charged impurities then the shape of $\rho(E)$ can change with pressure or with temperature. However, in the case of high compensation (which seems to be the case in our InGaP samples) the number of ionized impurities does not change substantially with pressure or with temperature. The changes of the free-carrier screening should not be very effective either. Another broadening mechanism which may occur is the hybridization of resonant states with the continuum of band states. For localized impurity states described by the Koster-Slater model [2] we obtain the Lorentzian broadening proportional to the density of band states at the impurity level $E_{\mathrm{d}}$. Such a hybridization does not occur for DX states coupled to the lattice.

Let us now apply Eq. (3) (without the second term) to our experimental results shown in Fig. 1. Instead of differentiating the experimental $n(P)$ it is better to fit the experimental points with a smooth curve (third-order polynomial fits very well) and differentiate this curve. For each $n(P)$ we obtain $E_{\mathrm{F}}(P)$ from the Fermi integral with $m_{\mathrm{eff}}(P)=0.1 m_{0}(1+8 P / 1900)$. Thus we neglect nonparabolicity and we take the effective mass to be proportional to the gap; the gap increases with 


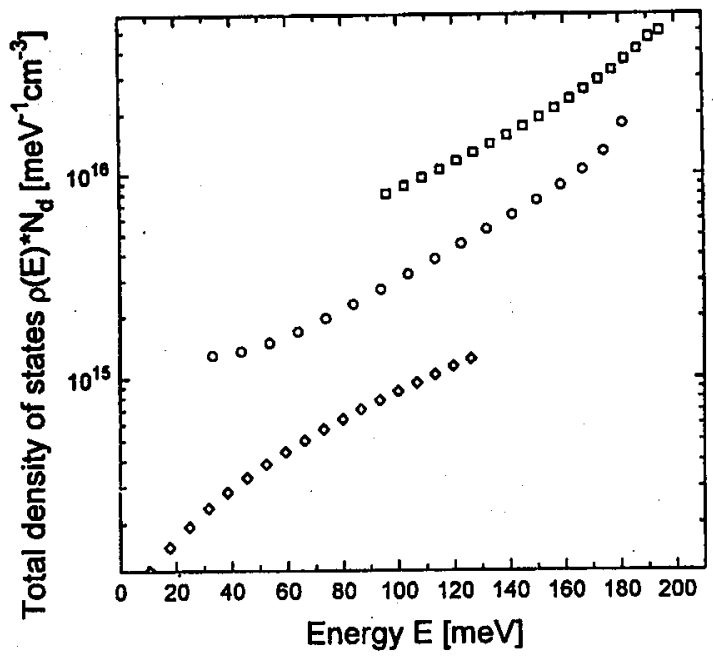

Fig. 2. Total density of resonant states in the conduction band for three InGaP:Si samples (in a log scale) as a function of energy (measured from the bottom of the conduction band). Symbols for different samples as in Fig. 1.

pressure from $1900 \mathrm{meV}$ with the coefficient $8 \mathrm{meV} / \mathrm{kbar}$. The only parameter which we have to insert is the pressure coefficient of the impurity level $\mathrm{d} E_{\mathrm{d}} / \mathrm{d} P$ (i.e., the pressure shift of $\rho(E)$ with respect to the conduction band edge). We always start from the pressure coefficient of the gap $(-8 \mathrm{meV} / \mathrm{kbar})$ because deep impurity states often do not move with respect to the valence band. When we plot $\mathrm{d} n / \mathrm{d} P$ divided by $\mathrm{d}\left(E_{\mathrm{d}}-E_{\mathrm{F}}\right) / \mathrm{d} P$ for the three samples we obtain three curves shown in Fig. 2 (we used the pressure coefficient $-10 \mathrm{meV} / \mathrm{kbar}$ which corresponds to that of the $X$ minimum). These curves should correspond to $N_{\mathrm{d}} \rho\left(E_{\mathrm{F}}-E_{\mathrm{d}}\right)$; they do not overlap because $N_{d}$ is different in each sample. When we multiply the lower-lying curves by appropriate factors we obtain a single curve corresponding to $N_{\mathrm{d}} \rho\left(E_{\mathrm{F}}-E_{\mathrm{d}}\right)$ in the highest-doped sample (Fig. 3). This is the final density of impurity states; it is clear that such a slowly-varying tail can be fitted with different curves. It is thus difficult to speculate about the position of the resonant Si level in InGaP from such data. However, in order to test our approximations we fitted the points in Fig. 3 by a Lorentzian with $E_{\mathrm{d}}=216 \mathrm{meV}$ (from the band-edge), $\Gamma=42 \mathrm{meV}$, and $N_{\mathrm{d}}=8.3 \times 10^{18} \mathrm{~cm}^{-3}$. Then we performed a standard analysis of our Hall data shown in Fig. 1. The fit was good. An equally good fit can be obtained with a different set of parameters: $E_{\mathrm{d}}=250 \mathrm{meV}, \Gamma=22 \mathrm{meV}$, $N_{\mathrm{d}}=2.7 \times 10^{19} \mathrm{~cm}^{-3}$. This illustrates our message that the experiment is only sensitive to $\rho(E)$ around the Fermi level and the extrapolations of $\rho(E)$ higher in the band can be misleading.

The concentration of acceptors can be adjusted to obtain the appropriate values of $n$ at $P=0$. The values which we listed in the caption to Fig. 1 indicate a rather high compensation in all three samples and are in agreement with the values of electron mobility. 


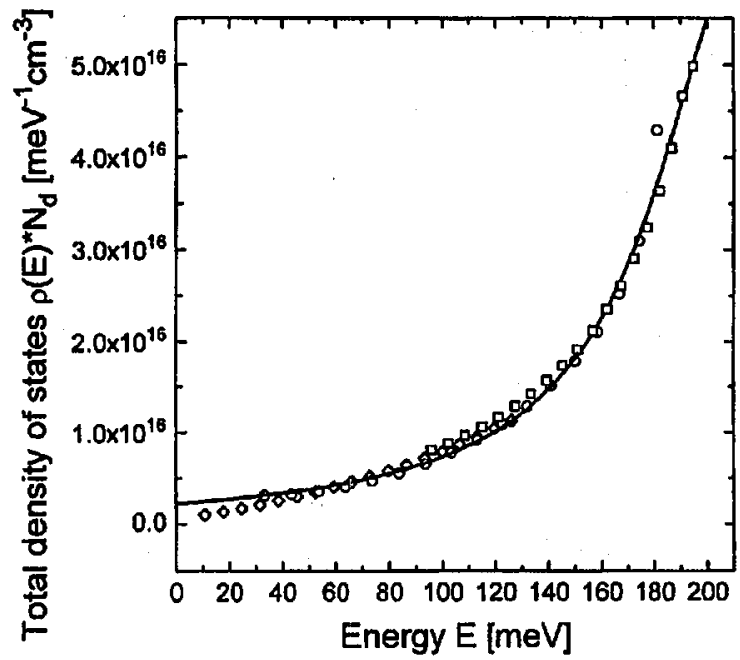

Fig. 3. Total density of resonant states for the highest-doped sample (linear scale) obtained from Fig. 2. Symbols for different samples as in Fig. 1.

It is worth noticing that similar $n(P)$ dependences have earlier been obtained in other materials (e.g. in GaAs:Si or in GaAs:Te) and our method can be applied to those results. High-temperature data can be interpreted with the second term in Eq. (3) included.

Let us finally speculate about the physical origin of the observed broad $\rho(E)$ distribution in our InGaP samples. X-ray diffraction data have shown that the composition fluctuation in the alloy does not exceed $1 \%$ which may give $10 \mathrm{meV}$ broadening of impurity states. Random potentials due to ionized impurities gave up to $40 \mathrm{meV}$ Gaussian broadening in GaAs:Si. The alloy splitting of impurity states could be similar to that in AlGaAs where it was shown to exceed $100 \mathrm{meV}$ [3]. This is the most probable source of broad distribution of $\rho(E)$ in our $\operatorname{In}_{0.5} \mathrm{Ga}_{0.5} \mathrm{P}$ samples.

The slowly varying tails of the impurity density of states lead to small temperature effects on $n$ while maintaining large pressure effects. This is very favorable for using such layers as pressure sensors.

This work was supported by the Committee for Scientific Research (Poland) through grant No. 8T 10C 02711.

\section{References}

[1] J.C. Zolper, H.C. Chui, Appl. Phys. Lett. 68, 3473 (1996).

[2] L. Konczewicz, W. Trzeciakowski, Phys. Status Solidi B 115, 359 (1983).

[3] R. Piotrzkowski, E. Litwin-Staszewska, P. Lorenzini, J.L. Robert, Semicond. Sci. Technol. 7, 103 (1992). 